\title{
Bilateral Prophylactic Mastectomy and Immediate Breast Reconstruction in High-Risk Women: The Importance of Health- Related Quality of Life in Decision Making
}

\author{
Valerie Lemaine, MD, MPH \\ Division of Plastic Surgery, Mayo Clinic, Rochester, MN
}

Women at elevated risk of breast cancer due to gene mutations or a strong family history of breast cancer are presented with several strategies to manage their breast cancer risk: enhanced screening, chemoprevention, and risk-reducing surgery. Women opting for enhanced screening may have yearly mammograms and magnetic resonance imaging along with clinical breast examinations. ${ }^{1}$ If chemoprevention is chosen as a means of lowering cancer risk, tamoxifen and raloxifene are two agents approved for this purpose in women at high risk of breast cancer who wish to avoid prophylactic surgery. Women selecting risk-reducing surgery will undergo bilateral prophylactic mastectomy (BPM), currently the most impactful approach in reducing cancer risk ${ }^{2-5}$ both in terms of the magnitude of the risk reduction it provides and in terms of its invasive and irreversible nature.

The article by McCarthy et al. published in this current edition of Annals of Surgical Oncology provides patientreported outcomes data and highlights the benefits of BPM and breast reconstruction on patient satisfaction and healthrelated quality of life in the first 2 years following surgery in this patient population. In this multisite study, McCarthy et al. sought to evaluate the impact of BPM and immediate breast reconstruction on health-related quality of life in high-risk women using several validated patient-reported outcomes instruments. The 1-year outcome analysis included 204 women, while for the 2-year follow-up, data from 149 women were available. In line with national

(C) Society of Surgical Oncology 2017

First Received: 14 May 2017;

Published Online: 9 June 2017

V. Lemaine, MD, MPH

e-mail: Lemaine.valerie@mayo.edu breast reconstruction trends, ${ }^{6}$ nearly two-thirds of women in this study underwent two-stage tissue expander/implant breast reconstruction. Autologous breast reconstruction was the second most frequent reconstructive method in this study (25.8\%), followed by a small proportion of direct-toimplant reconstructions (9.9\%). Study results show that 2 years following surgery, patient satisfaction with their reconstructed breasts was high. It is interesting to note that these women experienced higher psychosocial well-being, restored sexual well-being, and reduced anxiety within 2 years following surgery when compared with their preoperative baseline. Of significance, the two outcomes where patients scored worse compared with their preoperative baseline during the study period are physical wellbeing of the chest and upper body, and pain levels.

The value of this study is rooted in several factors, notably the multisite study design, the use of multiple validated patient-reported outcomes instruments, and the inclusion of baseline preoperative assessments that allow measurements of mean within-person changes of patientreported outcome scores. Several limitations are also found in Dr. McCarthy's study. First, the sample size is small and homogenous in terms of sociodemographic characteristics, consisting primarily of educated, high-income Caucasian women. Second, this study does not differentiate outcomes based on reconstructive method, and, lastly, follow-up duration is limited to 2 years, with a decreasing sample size for the 2-year outcome analysis.

A study by $\mathrm{Hu}$ et al. published in 2009 retrospectively compared long-term patient-reported outcomes between prosthetic and autologous breast reconstruction, using the BREAST-Q. ${ }^{7}$ In the first 5 years following surgery, patient satisfaction with their reconstructed breasts was high and similar for both reconstructive methods. Interestingly, beyond 5 years after surgery, patient satisfaction with their 
reconstructed breasts was greater among patients who underwent autologous breast reconstruction compared with tissue expander/implant procedures. A recently published study by Pusic et al. shows early results that corroborate these findings 1 year after mastectomy in a sample size of 1632 women. $^{8}$

Dr. McCarthy's present study provides high-quality information to guide management decisions for high-risk women looking to reduce their breast cancer risk. Decisions about prophylactic surgery require thoughtful consideration informed by adequate knowledge about the surgical procedures involved. In addition to the obvious benefit of cancer risk reduction, women at high-risk for breast cancer who are considering risk-reducing surgery should also take into account the impact of surgery on health-related quality of life and the risk of surgical complications.

Lee et al. recently published a cross-sectional survey study assessing decisions regarding postmastectomy breast reconstruction. ${ }^{9}$ In this study performed in a single academic center, women at high risk for breast cancer seeking prophylactic mastectomy were surveyed, along with women diagnosed with breast cancer and undergoing therapeutic mastectomy. In total, 126 women were included, with a survey response rate of $72 \%$. Two-thirds of the survey participants preferred to have mastectomy alone without reconstruction, yet less than half of these women made a decision aligned with that preference. Among the participants who preferred to undergo mastectomy with reconstruction, nearly $88 \%$ of them did so. Overall, the authors found that more than half of women received a surgical treatment that did not match their preference.

Rates of BPM rose by $12 \%$ per year between 1998 and $2008^{10}$ without showing signs of deceleration in more recent years. Increased awareness and availability and use of genetic testing are some of the possible factors contributing to this trend. From a surgical standpoint, significant technical improvements in mastectomy with the adoption of nipple-sparing techniques, combined with the constant advances in breast reconstruction techniques and technology (with the introduction of acellular dermal matrices, improved prosthetic devices, and use of autologous fat grafting, intraoperative fluorescent perfusion assessment and the prepectoral breast reconstruction technique) all work in synergy to influence women's decision to pursue BPM and breast reconstruction. Research endeavors such as the Mastectomy Reconstruction Outcomes Consortium are key to gathering prospective, multicenter, patient-reported outcomes data to guide women's decisions regarding mastectomy and breast reconstruction. Capturing patient-reported outcomes data over longer follow-up periods, stratifying results by type of reconstruction, and expanding diversity of the patient population from a sociodemographic perspective should be the focus of future research efforts in this field.

DISCLOSURES Valerie Lemaine has no disclosures to declare.

\section{REFERENCES}

1. Saslow D, Boetes C, Burke W, et al. American Cancer Society guidelines for breast screening with MRI as an adjunct to mammography. CA Cancer J Clin. 2007;57:75-89.

2. Hartmann LC, Schaid DJ, Woods JE, et al. Efficacy of bilateral prophylactic mastectomy in women with a family history of breast cancer. $N$ Engl J Med. 1999;340:77-84.

3. Domchek SM, Friebel TM, Singer CF, et al. Association of riskreducing surgery in BRCA1 or BRCA2 mutation carriers with cancer risk and mortality. JAMA. 2010;304:967-5.

4. Rebbeck TR, Friebel T, Lynch HT, et al. Bilateral prophylactic mastectomy reduces breast cancer risk in BRCA1 and BRCA2 mutation carriers: the PROSE Study Group. J Clin Oncol. 2004;22:1055-62.

5. Meijers-Heijboer H, van Geel B, van Putten WL et al. Breast cancer after prophylactic bilateral mastectomy in women with a BRCA1 or BRCA2 mutation. N Engl J Med. 2001;345:159-64.

6. Albornoz CR, Bach PB, Mehrara BJ, et al. A paradigm shift in U.S. Breast reconstruction: increasing implant rates. Plast Reconstr Surg. 2013;131:15-23.

7. Hu ES, Pusic AL, Waljee JF, et al. Patient-reported aesthetic satisfaction with breast reconstruction during the long-term survivorship period. Plast Reconstr Surg. 2009;124:1-8.

8. Pusic AL, Matros E, Fine N, et al. Patient-reported outcomes 1 year after immediate breast reconstruction: results of the Mastectomy Reconstruction Outcomes Consortium Study. J Clin Oncol. Epub 27 Mar 2017; JCO2016699561.

9. Lee CN, Deal AM, Huh R, et al. Quality of patient decisions about breast reconstruction after mastectomy. JAMA Surg. doi:10.1001/jamasurg.2017.0977.

10. Cemal Y, Albornoz CR, Disa JJ, et al. A paradigm shift in U.S. breast reconstruction: part 2. The influence of changing mastectomy patterns on reconstructive rate and method. Plast Reconstr Surg. 2013;131:320e-6e. 